\title{
Emergence of carbapenem resistance in Enterobacteriaceae strains in Padua (Italy) from January 2008 to July 20 I 2: phenotypic and genotipic assays
}

\author{
Andrea Bartolini', Ilaria Frasson', Maria Angela Biasolo',2, Ettore De Canale', Tiziana \\ Tommasini', Caterina Boldrin', Lucia Rossi', Sara N. Richter ${ }^{1,2}$, Antonietta Cavallaro' \\ I Servizio di Microbiologia e Virologia Azienda Ospedaliera di Padova \\ 2 Dipartimento di Medicina Molecolare, Università di Padova, Padova
}

Key words: Carbapenem-resistance, Enterobacteriaceae, Klebsiella pneumoniae-carbapenemase, Metallo-ßLactamase

Resistenze emergenti ai carbapenemi in ceppi di Enterobacteriaceae nel periodo 2008-20I2: test fenotipici e genotipici

\section{SUMMARY}

Introduction: In the management of nosocomial infections, the emergence of carbapenem-resistance in Enterobacteriaceae strains is a matter of increasing concern. The aim of this study was to detect and actively survey the presence of these strains by using phenotypic and genotypic assays.

Methods: The screening of the strains with reduced carbapenem susceptibility was carried out using the automated Vitek@2 System (bioMérieux). Subsequently, we performed both different confirmation phoenotypic assays (Modified Hodge Test, Kirby-Bauer test, Etest ${ }^{\circledR}$ MBL MP/MPI, Etest ${ }^{\circledR}$ AmpC, Mueller-Hinton/cloxacillin test) and molecular identification tests ( PCR assays).

Results: All the screened carbapenemase-producing strains (on the bases of the phenotypic tests results) were confirmed to harbour $\beta$-lactamase genes (by PCR analysis): 460 strains were positive for the presence of the blaKPC and 6 for the presence of the blaVIM gene.

Conclusions: KPC-positive Klebsiella pneumoniae strains caused in our hospital,in the period 2008-2012, an outbreak of infection that was subsequently restricted with effective containment measures.

It is mandatory that all carbapenemase-producing species will be closely monitored in hospital setting, to contain the possible rapid spread of strains showing more expansion properties and virulence.

\section{INTRODUZIONE}

Poiché i carbapenemi rappresentano la scelta terapeutica di riferimento nel caso di infezioni invasive da enterobatteri multi-antibiotico-resistenti, l'emergere di ceppi di Enterobacteriaceae produttori di carbapenemasi costituisce un problema clinico rilevante nella gestione delle infezioni nosocomiali $(1,2,4)$. Inizialmente appannaggio delle Unità di Terapia Intensiva, la diffusione di ceppi resistenti ai carbapenemi si è successivamente estesa a tutti gli ambiti ospedalieri, fino a coinvolgere pazienti non ospedalizzati, con infezioni "acquisite in comunità"(2). Il principale meccanismo di carbapenemico-resistenza è rappresentato dall'acquisizione/espressione di nuove $\beta$-lattamasi, dotate di attività idrolitica nei confronti dei carbapenemi. Gli enzimi attualmente più diffusi sono costituiti dalle carbapenemasi di Classe A (quali la KPC “Klebsiella-pneumoniae-carbapenemase"), le carbapenemasi di Classe B o Metallo- $\beta$ Lattamasi (di tipo VIM e NDM) e le oxacillinasi di Classe D (OXA-48-like) $(1,3)$.

La specie batterica che più frequentemente esprime questi meccanismi è $K$. pneumoniae, seguita da Escherichia coli, Enterobacter cloacae e da altre specie (3).

Lo scopo di questo studio è stato identificare e monitorare la presenza, nel nostro ospedale, di ceppi produttori di carbapenemasi ciò tramite l'utilizzo di test fenotipici e genotipici $(3,4,5,6)$.

\section{METODI}

Lo studio è stato condotto presso 1'U.O.C. di Microbiologia e Virologia di Padova nel periodo Gennaio 2008 - Luglio 2012.

Il monitoraggio è stato eseguito sia sui campioni provenienti dai reparti ospedalieri che da quelli ambulatoriali di nostra afferenza.

\section{Corresponding author: Andrea Bartolini}

Servizio di Microbiologia e Virologia Azienda Ospedaliera di Padova,

Via Giustiniani 2, 35I28 Padova - Tel 049 82I305 - Fax 04982 I 3054

E-mail: andbarto@libero.it 
Lo screening degli enterobatteri potenziali produttori di carbapenemasi è stato eseguito mediante l'utilizzo del sistema automatizzato Vitek®2 (bioMérieux). Sono stati selezionati gli isolati che presentavano diminuita sensibilità ad ertapenem, imipenem e meropenem (MIC $\geq 1 \mathrm{mg} / \mathrm{L}$ ).

Su tali ceppi sono stati eseguiti i seguenti test fenotipici di conferma:

1) Test di Hodge modificato, basato sulla riduzione dell'attività del carbapenemico saggiato nei confronti di un ceppo indicatore sensibile (ceppo di riferimento E. coli ATCC25922, controllo positivo $K$. pneumoniae ATCCBAA1705 , controllo negativo $K$. pneumoniae ATCCBAA-1706),

2) Antibiogramma secondo il metodo in disco-diffusione (Test di Kirby-Bauer),

3) Etest ${ }^{\circledR}$ MBL (MP/MPI) per la rivelazione di Metallo- $\beta$-Lattamasi,

4) Etest ${ }^{\circledR}$ AmpC e test su terreno Mueller-Hinton addizionato con cloxacillina per l'individuazione degli isolati produttori di $\beta$-lattamasi di tipo AmpC.

Inoltre sono stati eseguiti test genotipici di conferma con PCR per l'identificazione di carbapenemasi di tipo KPC, Metallo- $\beta$-Lattamasi (VIM e NDM), OXA-48-like e di $\beta$-lattamasi di tipo AmpC

\section{RISULTATI}

I ceppi positivi allo screening mediante Vitek ${ }^{\circledR} 2$ risultavano essere (Tabella 1):

K. pneumoniae: 7 ceppi nell'anno 2008, 33 ceppi nel 2009, 114 ceppi nel 2010, 174 ceppi nel 2011 e 130 ceppi nel 2012 (fino a Luglio compreso);

Enterobacter cloacae: 2 ceppi nell'anno 2011 ed 1 ceppo nel 2012 (fino a Luglio compreso);

Escherichia coli: 1 ceppo nell'anno 2010 ed 1 ceppo nel 2012 (fino a Luglio compreso);

Enterobacter aerogenes: 2 ceppi nell'anno 2012 (fino a Luglio compreso).

Gli isolati risultati positivi ai test fenotipici di conferma e successivamente identificati albergare geni di resistenza per i carbapenemi mediante PCR risultavano essere:

KPC-produttori: 460 ceppi (di cui 458 ceppi di $K$. pneumoniae e 2 ceppi di E. coli);

VIM-produttori: 6 ceppi (di cui 3 ceppi di $K$. pneumoniae e 3 ceppi di E. cloacae).

È da segnalare inoltre che 3 ceppi di K. pneumoniae albergavano sia geni per la produzione di carbapenemasi di tipo KPC che di tipo VIM. Non sono risultati presenti isolati positivi per determinanti di tipo NDM né di tipo OXA-48-like.

I ceppi di E. aerogenes non sono risultati carbapenemasi-produttori; la diminuita sensibilità ai carbapenemi è stata attribuita alla produzione di $\beta$-lattamasi di tipo AmpC ed alla concomitante alterazione delle porine.

\section{CONCLUSIONI}

Isolati clinici di $K$. pneumoniae KPC-positivi sono risultati responsabili di un outbreak ospedaliero che è stato successivamente limitato con misure di contenimento efficaci. Le altre specie di Enterobacteriaceae risultate positive per la presenza di determinanti di tipo KPC e VIM hanno prodotto un numero di infezioni contenute. Risulta tuttavia importante attuare una attenta sorveglianza che comprenda tutte le specie carbapenemico-resistenti, al fine di contenere l'eventuale rapida diffusione di ceppi con spiccate caratteristiche di diffusione e virulenza.

Esperienze in varie Aziende Ospedaliere hanno dimostrato come eradicare o contenere fortemente la diffusione delle infezioni sia possibile solo attraverso interventi di controllo aggressivi e mirati ad individuare tempestivamente le infezioni clinicamente manifeste ed i pazienti colonizzati.

Ciò permette di applicare in modo tempestivo le idonee misure di contenimento della diffusione (isolamento dei pazienti, igiene delle mani da parte degli operatori sanitari, dei pazienti e dei visitatori, pulizia e decontaminazione ambientale ecc...).

Con l'obiettivo di poter efficientemente contenere l'emergenza di focolai ospedalieri, sono state

Tabella I. Numero delle differenti specie di enterobatteri con MIC $\geq I \mathrm{mg} / \mathrm{L}$ per ertapenem, imipenem e meropenem mediante sistema automatizzato Vitek®2 (bioMérieux) nel periodo 2008-20I 2.

\begin{tabular}{|c|c|c|c|c|}
\hline \multirow[t]{2}{*}{ ANNO } & \multicolumn{4}{|c|}{ SPECIE } \\
\hline & KLPN & ENCL & ESCO & ENAE \\
\hline 2008 & 7 & 0 & 0 & 0 \\
\hline 2009 & 33 & 0 & 0 & 0 \\
\hline 2010 & 114 & 0 & $\mathrm{I}$ & 0 \\
\hline 2011 & 174 & 2 & 0 & 0 \\
\hline 20I2* & 130 & $\mathrm{I}$ & $\mathrm{I}$ & 2 \\
\hline TOTALE & 458 & 3 & 2 & 2 \\
\hline
\end{tabular}

Legenda. KLPN: Klebsiella pneumoniae; ENCL: Enterobacter cloacae; ESCO: Escherichia coli, ENAE: Enterobacter aerogenes.

*fino a Luglio 2012 compreso. 
recentemente messe a punto numerose linee guida Nazionali ed Internazionali.

Il Laboratorio di Microbiologia deve perciò acquisire dimestichezza nel riconoscimento di tali ceppi mediante l'uso appropriato e combinato di multipli test fenotipici e, ove possibile e necessario, completare la caratterizzazione con ricerche genotipiche.

\section{BIBLIOGRAFIA}

1. Nordmann P, Naas T, Poirel L. Global spread of Carbapenemase-producing Enterobacteriaceae. Emerg Infect Dis 2011; 17 (10): 1791-8.

2. Gaibani P, Ambretti S, Berlingeri A, et al. Rapid increase of carbapenemase-producing Klebsiella pneumoniae strains in a large Italian hospital: surveillance period 1 March-30 September 2010. Euro
Surveill 2011; 16 (8).

3. Luzzaro F, Pagani L, Rossolini GM, Sarti M, Stefani $\mathrm{S}$, Varaldo PE. Indicazioni per la conferma fenotipica della produzione di carbapenemasi nelle Enterobacteriaceae. Comitato di Studio AMCLI per gli Antimicrobici (CoSA). AMCLI 2012.

4. Gagliotti G, Cappelli V, Carretto E, et al. Indicazioni pratiche e protocolli operativi per la diagnosi, la sorveglianza e il controllo degli enterobatteri produttori di carbapenemasi nelle strutture sanitarie e socio-sanitarie. Azienda sanitaria e sociale regionale EmiliaRomagna 2011.

5. Richter SN, Frasson I, Biasolo MA, Bartolini A, Cavallaro C, Palu G. Ultrarapid detection of blaKPC $\mathrm{C}_{1 / 2-12}$ from perirectal and nasal swabs by use of real-time PCR. J Clin Microbiol 2012; 50 (5): 1718-20.

6. Doyle D, Peirano G, Lascols C, Lloyd T, Church DL, Pitout JD. Laboratory detection of enterobacteriaceae that produce carbapenemases. J Clin Microbiol 2012; 50 (12): 3877-80. 